\title{
Flächensparen in der Planung von Logistikimmobilien
}

\author{
Dennis Kotzold ${ }^{1}$ (D) $\cdot$ Lucas Hüer² $^{2}$ Kai-Michael Griese ${ }^{1} \cdot$ Martin Franz $^{3}$
}

Eingegangen: 25. Januar 2021 / Überarbeitet: 27. Juli 2021 / Angenommen: 3. August 2021 / Online publiziert: 18. Oktober 2021

(c) Der/die Autor(en) 2021

\section{Zusammenfassung}

Logistikunternehmen haben es bei der Suche nach neuen Standorten zunehmend schwerer, da geeignete Standorte immer knapper werden und ihnen wachsende Widerstände entgegenstehen: Umweltverbände und Anwohner*innen kritisieren Luftverschmutzung, Flächenverbrauch, Beeinträchtigungen des Landschaftsbilds und Zunahme des Verkehrslärms; die Lokalpolitik bevorzugt häufig Unternehmen mit höheren Gewerbesteueraufkommen und mehr (hochqualifizierten) Arbeitsplätzen. Der Druck auf die Logistikbranche, nachhaltigere Konzepte der Flächennutzung zu entwickeln und umzusetzen steigt. Zielsetzung des Artikels ist es, Ansätze aufzuzeigen, wie die Flächennutzung in der Logistik optimiert werden kann. Ein wichtiges Lösungsfeld ist auf Basis des Logistikmodells von Pfohl (2010) vor allem in den Bereichen der Flächenplanung zu verorten. Als Grundlage des Artikels wurde eine systematische Literaturrecherche durchgeführt. Die Ergebnisse zeigen Möglichkeiten auf, wie Unternehmen und Kommunen durch eine frühzeitige Planung zur Optimierung von Flächennutzung für logistische Prozesse beitragen können. Die sorgfältige Planung eines Lagers und die damit einhergehende Nutzung von effizienten Lagersystemen, verringern die benötigte Fläche und sorgen für verbesserte Prozesse im Bereich der Lagerund Umschlagslogistik. Auch lassen sich ggf. neue Lagerflächen vermeiden, wenn die bereits genutzten Flächen optimiert werden.

Schlüsselwörter Logistik · Flächennutzung · Digitalisierung · Unternehmenskooperation · Flächenplanung

\begin{abstract}
Logistics companies are finding it increasingly difficult to find new locations, as suitable sites are becoming scarcer and they are facing growing opposition: Environmental associations and local residents criticize air pollution, land consumption, negative impacts on the landscape and an increase in traffic noise; local politicians often prefer companies with higher business tax revenues and more (highly qualified) jobs. The pressure on the logistics industry to develop and implement more sustainable land use concepts is increasing. The objective of this article is to show approaches to how land use in logistics can be optimized. Mainly based on Pfohl's (2010) logistics model, an important field for a solution is to be found in areas of space planning. For the basis of the article, a systematic literature research was carried out. The results show the possibilities of companies and municipalities being able to contribute to the optimization of land use for logistics processes through early planning. Careful planning of a warehouse and the associated use of efficient storage systems reduce the amount of space required and ensure improved processes in the area of warehouse and handling logistics. New warehouse space may also be avoided if the space already in use is optimized.
\end{abstract}

Keywords Logistics $\cdot$ Land use $\cdot$ Digitization $\cdot$ Business cooperation $\cdot$ Land planning

Dennis Kotzold

d.kotzold@hs-osnabrueck.de

1 Fakultät Wirtschafts- und Sozialwissenschaften, Hochschule Osnabrück, Caprivistraße 30A, 49076 Osnabrück,

Deutschland
2 Fakultät Ingenieurwissenschaften und Informatik, Hochschule Osnabrück, Albrechtstraße 30, 49076 Osnabrück, Deutschland

3 Institut für Geographie, Universität Osnabrück, Seminarstr. 19 a/b, 49074 Osnabrück, Deutschland 


\section{Einleitung}

Logistikunternehmen haben es bei der Suche nach neuen Standorten oft schwer - gute Standorte sind knapp, Umweltverbände kritisieren Luftverschmutzung und Flächenverbrauch, Anwohner*innen kämpfen gegen Beeinträchtigungen des Landschaftsbilds und gegen den Verkehrslärm, und die Lokalpolitik vieler Kommunen bevorzugt Unternehmen mit mehr (hoch qualifizierten) Arbeitsplätzen und höheren Gewerbesteueraufkommen. Der Druck auf die Logistikbranche, flächensparende Konzepte zu entwickeln und umzusetzen, steigt. Generell ist es bisher nicht gelungen, den Flächenbedarf der Logistikbranche mit Nachhaltigkeitszielen - wie dem Ziel der Bundesregierung bis 2030 die Neuinanspruchnahme von Flächen für Siedlungen und Verkehr auf unter $30 \mathrm{ha} / \mathrm{Tag}$ zu verringern oder dem Sustainable Development Goal (SDG) 15.3 ,neutrale Landnutzung" (0 ha zusätzliche Versiegelung bis 2030) - in Einklang zu bringen (siehe Abb. 1). Der Flächenbedarf der Logistik nimmt immer weiter zu (siehe Abb. 2).

Trotzdem wird die Betrachtung der Logistik in Bezug auf die Flächeneffizienz bislang mit wenigen Ausnahmen (z.B. Wagner 2009) weitgehend vernachlässigt. Ziel des vorliegenden Beitrags ist es daher, Ansätze aufzuzeigen, wie die Flächennutzung in der Logistik optimiert werden kann. Als theoretischer Bezugsrahmen für die systematische Darstellung der Optimierungsansätze wird auf das Modell von Pfohl (2010) zurückgegriffen. Das Modell grenzt logistische Systeme im Unternehmen ab und erlaubt so eine logistikspezifische Betrachtung von Flächennutzung innerhalb eines Unternehmens. Ausgangspunkte sind nach Pfohl (2010) logistische Kosten bzw. Produktionsfaktoren, die als Input in die Logistikproduktion einfließen. Jeder Input wird in einem bestimmten Subsystem oder dem Logistiksystem selbst verarbeitet, wodurch eine logistische Leistung (der Output) entsteht. $\mathrm{Zu}$ diesen Subsystemen gehören z. B. ein Transportsystem, ein Auftragsbearbeitungssystem, ein La-

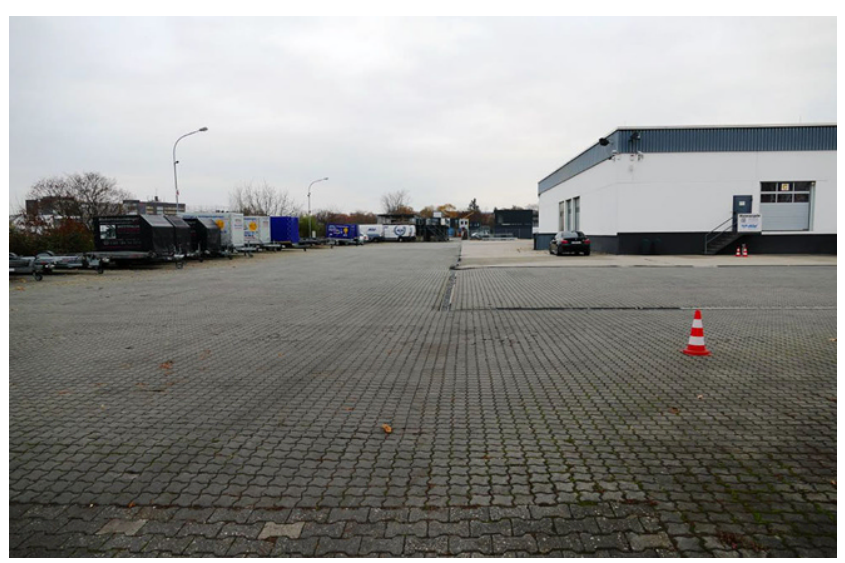

Abb. 1 Der Flächenbedarf der Logistik ist hoch und oft werden die Flächen nicht optimal genutzt. (Foto: Martin Franz) gersystem und ein Verpackungssystem. Abb. 3 zeigt dieses Modell in stark vereinfachter Form.

Im folgenden Kapitel wird das methodische Vorgehen erläutert. Anschließend werden die identifizierten Flächenoptimierungspotenziale am Beispiel der Inputphase mit Applikation für die Throughput und Outputphase dargestellt. Schließlich wird ein Fazit gezogen.

\section{Literaturanalyse}

Um eine fundierte Grundlage wissenschaftlicher Erkenntnisse zu gewinnen, wurde eine systematische Literaturrecherche für den Zeitraum 2000-2020 nach Webster und Watson (2002) durchgeführt. Dabei wurden die Suchbegriffe „logistics“, „land“, „land use“, „land optimisation“, „land management“, „,soil protection“, ,,cooperation“, ,,synergy“, ,digitisation“ und „supply chain“ verwendet. Dies führte zu einer Anzahl von 1547 Artikeln. Nach einer ersten Analyse, basierend auf dem jeweiligen Titel und Abstract, wurden 1136 irrelevante Publikationen entfernt, was zu 411 verbliebenen Quellen für die weitere Analyse führt. Dieser Aussortierungsschritt wurde an die Methode des PrismaModells (Moher et al. 2009) angelehnt und beachtet Kriterien wie Qualität, Aktualität, Themenbezug und Methodik der Artikel. Nach einer Prüfung der gesamten verbliebenen Publikationen auf Relevanz sowie einer zusätzlichen Vorwärts- und Rückwärtssuche werden 83 Veröffentlichungen als relevant für das weitere Vorgehen eingestuft (siehe Abb. 4).

Aufgrund der Längenbeschränkungen des vorliegenden Beitrags wird auf einen Einbezug der gesamten analysierten Literatur verzichtet. Stattdessen werden für die aus der Literaturanalyse abgeleiteten Handlungsbereiche nur die - aus Sicht der Autoren - wichtigsten Quellen, die sich der Flächenplanung im Rahmen der Inputphase widmen, erläutert. Unter Flächenplanung zur optimierten Flächennutzung verstehen wir die Entwurfserstellung einer, unter den gegebenen Voraussetzungen, bestmöglichen Auslastung und Nutzung der bestehenden und geplanten Logistikflächen, bei gleichzeitiger Beachtung ökonomischer, ökologischer und sozialer Ziele. Im Folgenden wird erläutert, wie effiziente Flächennutzungen durch Anpassungen in der Planung erreicht werden können und wie sich diese in das Modell von Pfohl (2010) einordnen lassen.

\section{Ergebnisse zur Inputphase (Logistikkosten) - effektive Flächennutzung durch frühzeitige Planung}

Eine wichtige Grundlage, um die Auslastung und Nutzung logistischer Flächen zu optimieren, entsteht bereits bei der Flächenplanung vor der Inbetriebnahme. Die Bedeutung 
Abb. 2 Entwicklung des Lager- und Logistikflächenumsatzes (Gesamtsumme aller Flächen, die vermietet, verkauft oder verleast worden sind) in Deutschland im Zeitraum von 2003-2019. (BNP Paribas Real Estate GmbH 2013, S. 4, 2020, S. 4) duktion von logistischen Dienstleistungen. (in Anlehnung an Pfohl 2010)
Abb. 3 Grundstruktur der Pro-

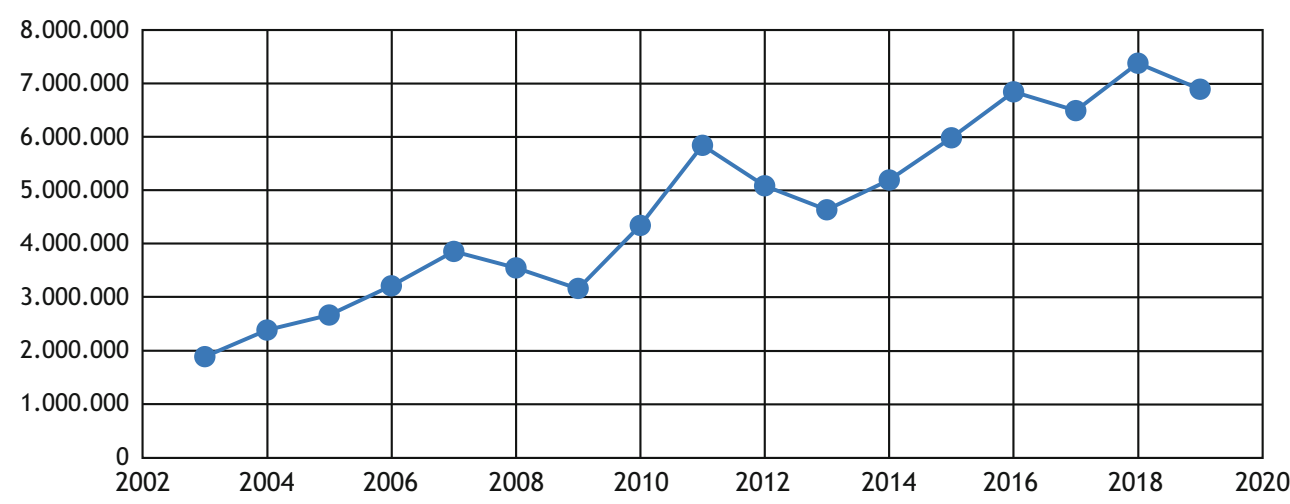

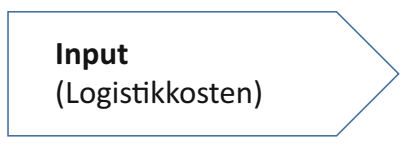

Produktionsfaktoren

(Arbeit, Betriebsmittel, Material einschließlich Energie, Informationen)

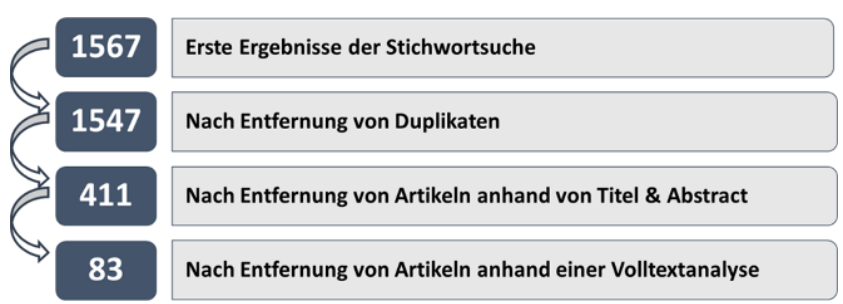

Abb. 4 Ergebnisse der systematischen Literaturrecherche

dieser Inputphase ist mit der Tatsache zu begründen, dass in der Planung das Fundament für alle weiteren Optimierungsmöglichkeiten des Throughput (Logistikproduktion) und des Outputs (Logistikleistung) liegt. In der Literatur zeigten sich zur Flächenplanung unterschiedliche Bezugspunkte.

\section{Flächenplanung in Kommunen}

Erste Ansatzpunkte für die effektive Flächennutzung zeigen sich in der Planungsphase von Logistikflächen aus Perspektive der Kommunen bzw. der verantwortlichen kommunalen Vertreter. Für die Logistikbranche gelten bei der Neuansiedelung oftmals gesonderte rechtliche, zeitliche (24-Stunden-Betrieb) und räumliche (Mindestabstand zur Wohnbebauung) Kriterien, wodurch viele Flächen ungeeignet sind (Pyschny et al. 2012). Neben der bevorzugten Ausweisung von Brachflächen und der Erschließung entsprechender Potenziale durch ein Flächenmanagement besteht die Möglichkeit, über Vorgaben im Flächennutzungsplan, Bebau-

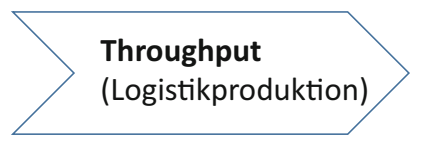

Logistiksystem

(Produktionssystem der logistischen Leistung)

(Auftragsabwicklung, Bestandsmanagement, Verpackung, Transport, Lagerung)

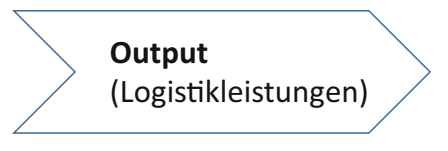

Versorgungs-

Lieferservice

(das richtige Gut im richtigen Zustand, zur richtigen Zeit am richtigen Ort) ungsplan und Gestaltungssatzungen Einfluss auf die Gestaltung neuer Logistikflächen zu nehmen. Da geeignete Flächen in vielen Regionen knapp geworden sind, können Kommunen dabei durchaus selbstbewusst auftreten und müssen ggf. auch auf eine Flächennutzung verzichten, um Ökosysteme und damit verbundene Lebensräume nicht $\mathrm{zu}$ gefährden. Auch müssen Kompensationsflächen und Maßnahmen zur ökologischen Aufwertung der Fläche (z. B. Bepflanzung, Dachbegrünung) bei der Planung bedacht werden. Um den Flächenwettbewerb zu vermeiden, erscheint es hilfreich, wenn Vorgaben auf regionaler Ebene zwischen den Kommunen vereinheitlicht sind. Zusätzliche Einflussmöglichkeiten haben die Kommunen, wenn sie selbst im Besitz der Flächen sind (Pyschny et al. 2012; Bundesamt für Bauwesen und Raumordnung 2001).

\section{Flächenplanung im Unternehmen}

Auch aus Perspektive der Unternehmen bzw. der verantwortlichen Entscheider*innen im Unternehmen zeigen sich im Rahmen ihrer Flächenplanung umfassende kurz, mittel- sowie langfristige Möglichkeiten zur Optimierung der Auslastung und Nutzung logistischer Flächen. Unlängst ist das „Streben nach ökoeffizienten Lösungen ein permanenter Bestandteil logistischer Betrachtungen" geworden, sodass Ökoeffizienz durch eine Optimierung von „Materialeinsatz, Energieverbrauch und Flächenbelegung“ erreicht werden soll (Deckert 2016, S. VIII). Neben der allgemein sinnvollen, mehrstöckigen Bauweise, sollte darauf geachtet werden, dass Pufferräume vorhanden sind, eine flexible Tei- 
lung der Räume in unterschiedlich große Flächen möglich ist und Potenziale für Anbauten oder Aufbauten existieren. Bei der Planung der Bauweise sollte dementsprechend auf eine modulare und ausbaufähige Gebäudestruktur geachtet werden (Kujath 2003; Förster et al. 2017). Produzierende Unternehmen haben in Bezug auf ihre Logistikflächen vor allem durch die geplante Produktionsstruktur (z. B. just in time) sowie die Materialflussstruktur einen großen Einfluss auf Flächen- und Raumnutzungen, indem Durchlaufzeiten, Lagerbestände und die Flexibilität der Auftragsabwicklung beachtet werden (Pawellek 2007). Weitere Möglichkeiten, um Flächeneinsparungen in der Lagerlogistik zu erzielen, liegen gleichermaßen im Lagerlayout und der Lagertechnologie, wie auch in den einzulagernden Gütern (Vahrenkamp und Kotzab 2012; Zowada und Niestrój 2019). Auf der einen Seite kann durch eine optimierte Flächenplanung die Grundfläche optimal ausgenutzt werden, z. B. durch ein Hochregallagersystem oder die Minimierung der Gangbreiten (Deckert 2016). Auf der anderen Seite können einzulagernde Güter durch ein einheitliches Verpackungsdesign so gestaltet werden, dass eine effektivere Nutzung des vorhandenen Platzes möglich ist (Zowada und Niestrój 2019).

\section{Flächenplanung für Mehrzweck- und Nachnutzung}

Weitere Möglichkeiten bestehen, indem die bebauten Flächen als Mehrzwecklösung für verschiedene Stakeholder geplant werden, um so die Auslastung der Flächen zu optimieren (Haag 2006). Eine solche Mehrzwecknutzung kann z. B. durch eine Kooperation mit Lieferanten oder Kunden aus der gleichen Lieferkette erfolgen und einen kleinen, mittleren oder größeren Flächeneffekt beinhalten. Neue Informations- und Kommunikationstechnologien (z. B. Sensoren, Big Data, KI) können hier eine Informationsbasis schaffen, die die Koordination zwischen unterschiedlichen Akteuren erleichtern (z. B. Lenzbauer 2007). Aber auch der Einsatz der Fläche zu vollkommen anderen Zwecken wie Großveranstaltungen ist denkbar, auch wenn dies bei Logistikgebäuden zu Anfang meistens nicht möglich erscheint. Ein zusätzlicher - und im besten Fall gleichzeitiger - Nutzen kann sich auch positiv auf die regionale Akzeptanz durch die Bevölkerung auswirken. Die Nutzung für Veranstaltungen, die viel Platz in Anspruch nehmen und mit Lärm verbunden sind, und somit im Innenstadtleben oft als störend empfunden werden, können in die Planung des Gebäudes bzw. der Außenflächen mit einfließen. Exemplarisch sei das Parkhausdach Moffet Place High Gardens in Redwood (USA) genannt, bei dem mehrere Sportplätze auf dem Dach installiert sind. Außerdem sollten Gebäude so errichtet werden, dass sie nach der Nutzung für Logistikprozesse noch zu anderen Zwecken eingesetzt werden können (Förster et al. 2017).

\section{Flächenplanung und Kosten}

Im Bereich der Lagerlogistik kann bei vielen Unternehmen ein Trend hin zur Dezentralisierung beobachtet werden (Vahrenkamp und Kotzab 2012). Dies bedeutet, dass immer mehr regionale Lagerhäuser und Distributionszentren errichtet werden, um die Summe der Absatzwege zu den Abnehmern zu senken und somit Transportkosten und -zeiten zu verringern. Dadurch müssen immer mehr Flächen zur Verfügung stehen, um eine regionale Lagerung zu gewährleisten. Dies ist nicht nur aus ökologischen (mehr Bodenversiegelung etc.) und sozialen (Flächenverteilungskonflikte etc.) Gesichtspunkten, sondern auch aus ökonomischen Gründen nicht immer sinnvoll. Die Gesamtkosten eines Distributionssystems steigen ab einer zu großen Anzahl an Lagerflächen rapide an (Vahrenkamp und Kotzab 2012). Auch wenn die Errichtung zusätzlicher Lager zu Beginn für deutlich geringere Transportkosten sorgt, da die Entfernungen zum Kunden geringer sind, steigen die Transportkosten wieder an, sobald die Auffüllmengen der regionalen Lager $\mathrm{zu}$ gering und damit unwirtschaftlich werden. Außerdem müssen Transportmöglichkeiten für die einzelnen Regionen geschaffen werden, was die Flotte und somit auch den Verkehr und die Parkfläche vergrößern würde. Da zusätzlich die Lagerkosten ansteigen, wenn mehr Lagerhäuser gebaut oder gemietet werden, wird eine zu große Anzahl an verschiedenen regionalen Lagern schnell unwirtschaftlich (siehe Abb. 5). Dies zeigt, dass neben sozialen und ökologischen Problemen oftmals auch ökonomische Konsequenzen entstehen, wenn Flächen nicht effizient ausgelastet werden.

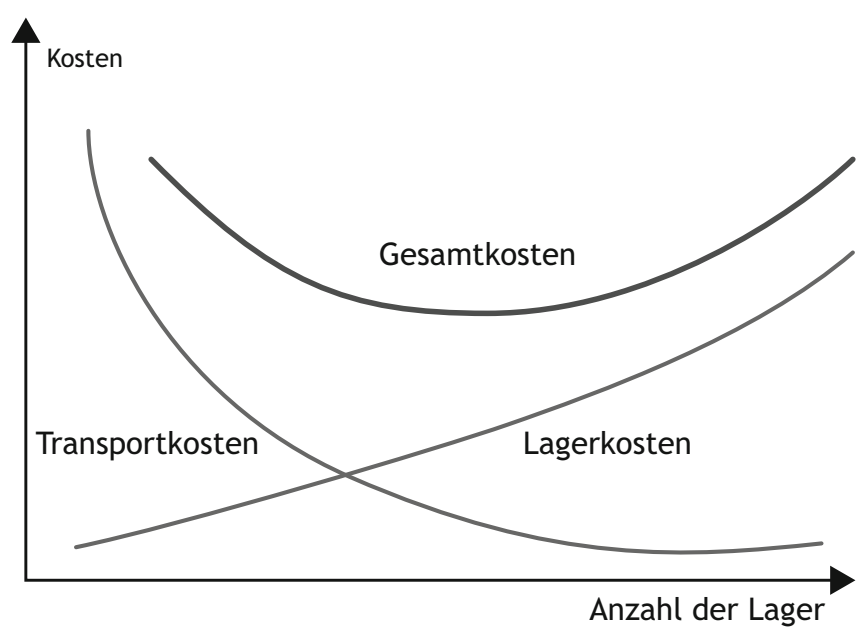

Abb. 5 Kostenstruktur von Distributionssystemen. (Vahrenkamp und Kotzab 2012) 
Tab. 1 Merkmale einer effizienten und systematischen Flächenplanung

\begin{tabular}{lll}
\hline $\begin{array}{l}\text { Merkmale der effizien- } \\
\text { ten Flächenplanung }\end{array}$ & Fragen zur Ermittlung & Beispiele \\
\hline Entscheidungsträger & Wer ist verantwortlich? & Kommunen, Unternehmer*in, Stakeholder \\
Zeitraum & Wie lang ist der Planungszeitraum? & Kurzfristig, mittelfristig, langfristig \\
Umfang & Wie groß ist der Flächeneffekt? & Klein, mittel, groß \\
Kosten & Wie hoch sind die Kosten für die effiziente Planung \& Realisierung? & Gering, mittel, hoch \\
Mehrzwecknutzung & Wie können die Flächen für weitere Stakeholder genutzt werden? & Großveranstaltungen \\
$\begin{array}{l}\text { Digitalisierung } \\
\text { Flexibilität }\end{array}$ & Welche Technologien kommen zum Einsatz? & Sensorik, Big Data/KI \\
Verzicht & Wie lassen sich die Räume für eine vielseitige Nutzung gestalten? & Mehrstöckige Bauweise, Hochregallagerung \\
Produktion & Auf was kann ggf. verrichtet werden? & Dezentrale Lager, extra Umschlagsfläche \\
\hline & Ist die Produktion auf die Flächennutzung abgestimmt? & Just-in-time-Produktion, flexible Lagerung \\
\hline
\end{tabular}

\section{Fazit}

Für Logistikunternehmen ist es schwieriger geworden, geeignete Flächen für die Ansiedlung zu finden und gleichzeitig müssen die verfügbaren Flächen umweltfreundlicher und effizienter genutzt werden. Dieser Artikel zeigt auf Basis einer Literaturanalyse, in welchem Rahmen die Flächennutzung für Logistikprozesse optimiert werden kann. In Anlehnung an das Modell von Pfohl (2010) zeigt dieser Beitrag, dass eine frühzeitige Optimierung der Flächenplanung im Rahmen der Inputphase eine wichtige Grundlage für die effiziente Flächennutzung als auch für die Throughputund Outputphasen ermöglicht. So kann z. B. die sorgfältige Planung (Input) eines Lagers und die damit einhergehende spätere Nutzung von effizienten Lagersystemen (z.B. Hochregallager) oder eines individuellen Lagerdesigns die benötigte Fläche verringern und gleichzeitig für verbesserte Prozesse im Bereich der Lager- und Umschlagslogistik sorgen. Dadurch lässt sich bei der Planung eines neuen Gebäudes Fläche einsparen. Tab. 1 zeigt zusammenfassend ausgewählte Merkmale und resultierende Fragen auf, die als Startpunkt für effiziente und systematische Flächenplanung von Bedeutung sind.

Da die Ergebnisse auf einer ersten Literaturanalyse basieren, sollten die Merkmale in zukünftigen wissenschaftlichen Arbeiten überprüft, validiert und ergänzt werden. Um die Flächennutzung auch nach den Planungsaktivitäten kontinuierlich zu verbessern und den Ausbau der bestehenden Logistikinfrastruktur zu vermeiden, erscheinen Kooperationen sinnvoll. Für die Realisierung von Kooperationen müssen jedoch viele Voraussetzungen gegeben sein (z.B. Nähe, Kooperationsbereitschaft), die im Vergleich zur Flächenplanung (Input) komplexer und schwieriger erscheinen (Hingley et al. 2011; de Souza et al. 2014; Pérez-Bernabeu et al. 2015). Auch mit der Digitalisierung von Logistikprozessen (z.B. über Sensorik) sind Flächeneinsparungen bei der Logistikproduktion denkbar (Lenzbauer 2007; Fürstenberg und Kirsch 2017). Diese Optimierungsmöglichkeiten müssen auch in Zukunft weiter wissenschaftlich untersucht werden. Diesbezüglich könnte die Anpassung des Modells von Pfohl (2010) eine gute Möglichkeit bieten.

Insgesamt gibt dieser Artikel einen ersten Überblick für Möglichkeiten zur Flächenreduzierung in der Logistik, um somit im Sinne des SDG 15.3. für eine nachhaltigere Flächenentwicklung von Logistikprozessen zu sorgen.

Funding Open Access funding enabled and organized by Projekt DEAL.

Open Access Dieser Artikel wird unter der Creative Commons Namensnennung 4.0 International Lizenz veröffentlicht, welche die Nutzung, Vervielfältigung, Bearbeitung, Verbreitung und Wiedergabe in jeglichem Medium und Format erlaubt, sofern Sie den/die ursprünglichen Autor(en) und die Quelle ordnungsgemäß nennen, einen Link zur Creative Commons Lizenz beifügen und angeben, ob Änderungen vorgenommen wurden.

Die in diesem Artikel enthaltenen Bilder und sonstiges Drittmaterial unterliegen ebenfalls der genannten Creative Commons Lizenz, sofern sich aus der Abbildungslegende nichts anderes ergibt. Sofern das betreffende Material nicht unter der genannten Creative Commons Lizenz steht und die betreffende Handlung nicht nach gesetzlichen Vorschriften erlaubt ist, ist für die oben aufgeführten Weiterverwendungen des Materials die Einwilligung des jeweiligen Rechteinhabers einzuholen.

Weitere Details zur Lizenz entnehmen Sie bitte der Lizenzinformation auf http://creativecommons.org/licenses/by/4.0/deed.de.

\section{Literatur}

BNP Paribas Real Estate GmbH (2013) Property Report. Logistikmarkt Deutschland 2013. www.iz-shop.de/buch-4800/logistikmar kt-deutschland-2013?barrier=false. Zugegriffen: 11. Jan. 2021

BNP Paribas Real Estate GmbH (2020) Logistikmarkt Deutschland. Property Report 2020. www.realestate.bnpparibas.de/sites/ default/files/document/2020-02/bnppre-logistikimmobilienmarktdeutschland-property-report-2020.pdf. Zugegriffen: 11. Jan. 2021

Bundesamt für Bauwesen und Raumordnung (2001) Auf dem Weg zu einer nachhaltigen Siedlungsentwicklung. http://www.kinderum weltgesundheit.de/index2/pdf/MEILENSTEINE/un-welt/2001_ ISTANBUL+5.pdf. Zugegriffen: 18. Febr. 2021 (Berlin)

Deckert C (Hrsg) (2016) CSR und Logistik: Spannungsfelder Green Logistics und City-Logistik, 1. Aufl. Management-Reihe Corporate Social Responsibility. Springer, Berlin, Heidelberg 
Förster A, Wenzel S, Thierstein A, Gilliard L, Scholze L, Unland L, Brunner B (2017) Gewerbe \& Stadt. Gemeinsam Zukunft gestalten. https://mediatum.ub.tum.de/doc/1398132/file.pdf. Zugegriffen: 18. Febr. 2021 (Technische Universität München)

Fürstenberg K, Kirsch C (2017) Intelligente Sensorik als Grundbaustein für cyber-physische Systeme in der Logistik. In: ten Hompel M, Vogel-Heuser B, Bauernhansl T (Hrsg) Springer Reference Technik. Handbuch Industrie 4.0, 2. Aufl. Springer Vieweg, Berlin, Heidelberg, S 271-297

Haag H (2006) Siedlungsflächenmanagement - Instrumente zur Steuerung der Siedlungsentwicklung. http://www.vlp-aspan.info/files/ papers/siedlungsflaechenmanagement.pdf. Zugegriffen: 18. Feb. 2021

Hingley M, Lindgreen A, Grant DB, Kane C (2011) Using fourth-party logistics management to improve horizontal collaboration among grocery retailers. Supply Chain Manag 16(5):316-327. https:// doi.org/10.1108/13598541111155839

Kujath HJ (2003) Logistik und Raum - Neue regionale Netzwerke der Güterverteilung und Logistik. https://leibniz-irs.de/fileadmin/ user_upload/IRS_Working_Paper/LogistikuRaum.pdf. Zugegriffen: 29. Dez. 2020 (Institut für Regionalentwicklung und Strukturplanung, Working Paper No. 18. Erkner)

Lenzbauer S (2007) RFID - Anwendungen in der Logistik. Schriftenreihe des Instituts für Transportwirtschaft und Logistik, Bd. Logistik, 02/2007. Institut für Transportwirtschaft und Logistik, Wirtschaftsuniversität Wien, Wien

Moher D, Liberati A, Tetzlaff J et al (2009) Preferred reporting items for systematic reviews and meta-analyses: the PRISMA statement. PLoS Med 6(7):e1000097
Pawellek G (2007) Produktionslogistik. Planung-Steuerung-Controlling. Carl Hanser, München

Pérez-Bernabeu E, Jua AA, Faulin J, Barrios BB (2015) Horizontal cooperation in road transportation: a case illustrating savings in distances and greenhouse gas emissions. Int Trans Oper Res 22(3):585-606. https://doi.org/10.1111/itor.12130

Pfohl HC (2010) Logistiksysteme. Springer, Berlin, Heidelberg

Pyschny A, Wilde E, Knippschild R (2012) Auf dem Weg zur Stadtregion? Raumforsch Raumordn 70(6):531-544

de Souza R, Goh M, Lau HC, Ng WS, Tan PS (2014) Collaborative urban logistics-synchronizing the last mile a Singapore research perspective. Procedia-social Behav Sci 125:422-431. https://doi. org/10.1016/J.SBSPRO.2014.01.1485

Vahrenkamp R, Kotzab H (2012) Logistik: Management und Strategien. Walter de Gruyter, Berlin

Wagner T (2009) Verkehrswirkungen von Logistikansiedlungen - Abschätzung und regionalplanerische Bewertung. Schriftenreihe des Instituts für Verkehrsplanung und Logistik - Harburger Berichte zur Verkehrsplanung und Logistik, Bd. Band 4. Technische Universität Hamburg-Harburg, Hamburg (Dissertation)

Webster J, Watson RT (2002) Analyzing the past to prepare for the future: Writing a literature review. MISQ 26(2):xiii-xxiii

Zowada K, Niestrój K (2019) Cooperation of small and medium-sized enterprises with other supply chain participants in implementing the concept of green logistics. Res Pap Wroclaw Univ Econ 63(6):249-260. https://doi.org/10.15611/pn.2019.6.20 